\title{
Rumo aos 40 anos de entrevista motivacional: evolução da abordagem
}

\author{
Flavia Serebrenic* \\ Danielle Ruiz Lima**
}

\begin{abstract}
Resumo
A Entrevista Motivacional (EM) é uma abordagem pautada na empatia, na escuta reflexiva e na resolução da ambivalência para auxiliar no processo de mudança. Desde sua criação, foram realizadas revisões teóricas importantes com o objetivo de aprimorar o método e melhor adequá-lo às diferentes demandas, porém a literatura no Brasil ainda encontra-se desatualizada. Método: Foi realizada uma revisão não-sistemática da literatura sobre a EM buscando sintetizar e apresentar as principais mudanças teóricas realizadas. Resultados e Discussão: Os princípios centrais e as fases da mudança foram algumas das reformulações propostas, que agora melhor refletem a complexidade do processo e oferecem diretrizes mais estruturadas para direcionar o trabalho do terapeuta, esclarecendo também alguns dos conceitos originais. Conclusões: Considerando o aumento da utilidade da EM nos diversos contextos e as mudanças realizadas ao longo dessas últimas décadas, acredita-se que esta atualização favorecerá o ensino, pesquisa e a prática da EM no país.

Palavras-chave: Entrevista Motivacional; motivação; mudança.
\end{abstract}

\section{Towards 40 years of motivational interviewing: evolvement of the approach}

\begin{abstract}
Motivational Interviewing (MI) is an approach that relies on empathy, reflexive listening and ambivalence resolution to assist people in the process of changing. Since its creation, important theoretical revisions have been made with the aim of improving the method according to different demands, but literature in Brazil is found to be out of date. Method: A non-systematic review of literature on MI was carried out, seeking to synthesize and present the main theoretical changes made. Results and Discussion: The main principals and phases of change were among some of the changes proposed, which now reflect the complexity of the process and provide more structured guidelines for the therapist, also clarifying some of the original concepts. Conclusions: Considering the increase of the MI utility in various contexts and changes made over the last decades, it is believed that this update will favor the teaching, research and practice of $\mathrm{MI}$ in the country.
\end{abstract}

Keywords: change; motivation; Motivational Interviewing.

* Psicóloga - PUCSP, Mestre pela Universidade de Londres e Doutora em Psiquiatria e Psicologia médica - UNIFESP. Colaboradora do Grupo Interdisciplinar de Estudos de Álcool e outras Drogas (GREA), do Instituto de Psiquiatria da Faculdade de Medicina da Universidade de São Paulo (IPq-FMUSP).

** Psicóloga, Mestre e Doutoranda em Ciências pela FMUSP. Colaboradora do Grupo Interdisciplinar de Estudos de Álcool e outras Drogas (GREA), do Instituto de Psiquiatria da Faculdade de Medicina da Universidade de São Paulo (IPq-FMUSP). 


\section{Introdução}

A Entrevista Motivacional (EM) foi criada com o objetivo de estimular a motivação para a mudança do comportamento do uso de substâncias há mais de três décadas, em uma época em que intervenções tradicionais estavam sendo questionadas dada a falta de evidências sobre sua eficácia e suspeitas de possíveis danos (White \& Miller, 2007). Estas abordagens tradicionais apoiavam-se na ideia de que as pessoas que sofriam com problemas com álcool e outras drogas estariam em negação, resistência, possuíam desvios de caráter ou mesmo transtornos de personalidade (Miller \& Rollnick, 1991).

Introduzida pelo psicólogo William R. Miller e posteriormente desenvolvida em parceria com Stephen Rollnick, a EM é definida como um método diretivo, centrado na pessoa, com foco no aumento da motivação intrínseca para a mudança por meio da resolução da ambivalência (Miller \& Rollnick, 1991).

Até hoje, foram publicadas três edições teóricas sobre a EM, porém apenas a primeira versão, publicada nos Estados Unidos em 1991, foi traduzida para o português e apenas 10 anos depois, em 2001. Desde a criação, o foco de trabalho da EM foi ampliado para outros tipos de mudanças (além do uso de substâncias) e importantes reformulações teóricas foram feitas. Além disso, questões sobre o treinamento e a qualidade da aplicação do método têm sido mais recentemente abordadas (Miller \& Rollnick, 2009). Considerando este cenário, estudos sobre a EM precisam acompanhar esta evolução para que sua prática seja adequadamente testada e validada. Da mesma forma, a atualização teórica da EM torna-se essencial para promover a disseminação da prática nas diversas áreas em que a abordagem pode ser útil.

O objetivo deste artigo, portanto, é apresentar a teoria da EM, ressaltando as principais mudanças pensadas pelos seus criadores até os dias atuais, considerando, principalmente, a importância de atualizar a literatura sobre o tema no Brasil.

\section{Surgimento da Entrevista Motivacional}

No início da década de 1980, o psicólogo William R. Miller passou a testar uma postura alternativa diante das dificuldades observadas nas pessoas que ele atendia, utilizando uma escuta diferenciada para entender e valorizar motivações individuais para a mudança de comportamento. A partir de alguns experimentos, Miller observou que o estilo empático do terapeuta se associou à melhora significativa do comportamento-problema quando comparado a técnicas comportamentais (Miller
\& Rose, 2009). Inúmeros estudos mais tarde vieram confirmar o efeito positivo do estilo do terapeuta nos resultados da intervenção (Moyers \& Rollnick, 2002; Miller \& Rose, 2009). Assim, em 1983, Miller formalizou alguns conceitos iniciais da abordagem, resultando no primeiro artigo sobre o tema (Miller, 1983; Miller \& Rose, 2009).

Além da empatia, Miller concluiu que um método eficaz para aumentar o comprometimento com a mudança precisaria também envolver estratégias que promovessem a argumentação, por parte da pessoa, a favor da ideia (no caso, a possibilidade de mudança). No entanto, práticas confrontativas - ainda hoje utilizadas em muitos contextos - fazem o contrário, pois exigem da pessoa a prontidão para a mudança com base na coerção, o que tende a fortalecer o status quo. Por outro lado, enquanto que no confronto a pessoa é forçada a reconhecer a necessidade de mudança, no aconselhamento simples, defendido como mais humanizado, o terapeuta a auxilia de maneira paternalista, não apresentando um direcionamento claro. Além disso, em ambos os casos o terapeuta é quem normalmente possui as respostas para o problema vivenciado pela pessoa. Dentro desta lógica, independentemente da postura do terapeuta, os tratamentos teriam alguma chance de sucesso apenas com pessoas que atingiam "O fundo do poço", pois estas estariam suficientemente motivadas (Miller,1983). Estas reflexões levaram Miller a diferenciar a EM das abordagens tradicionais, que seria especialmente útil para pessoas com problemas ainda mais brandos relacionados ao uso (ver tabela 1).

Pouco tempo depois, o psicólogo inglês Stephen Rollnick teve a oportunidade de compartilhar seu entusiasmo pessoalmente com Miller sobre os resultados observados com o uso da abordagem em pacientes com tabagismo (Miller \& Rose, 2009). Rollnick encorajou Miller a escrever mais sobre a nova abordagem, o que resultou em uma parceria entre os dois que duraria até os dias de hoje. A teoria da EM então foi consolidada com a publicação do primeiro livro sobre o tema, em 1991 (Miller \& Rollnick, 1991).

Vales ressaltar que na perspectiva da EM o termo cliente é preconizado, por este evitar colocar a pessoa na posição de incapaz, tendo como referência as abordagens humanistas de Carl Rogers (Rogers, 1951). Porém, considerando que a EM refere-se essencialmente à postura do profissional, defende-se aqui que, desde que o profissional transmita valorização e empoderamento da pessoa, a nomenclatura utilizada não deve ser um problema (Covell, McCorkle, Weismann, Summerfelt, \& Essock, 2007). 
Tabela 1. Principais diferenças entre as abordagens tradicionais e a Entrevista Motivacional para a mudança de comportamento

\begin{tabular}{lll}
\hline & Abordagens tradicionais & Entrevista Motivacional \\
\hline Quem é o "expert" & Terapeuta & Paciente/cliente/pessoa \\
Estilo & Confronto ou aconselhamento & Colaboração/parceria \\
Cliente & Passivo & Ativo \\
Duração da intervenção & Quanto mais longa mais efetiva & Breve pode ser efetiva \\
O que prevalece & Autoridade/saber do profissional & Autonomia/saber da pessoa \\
Motivação & Pessoa deve estar pronta para agir & Dinâmica, pode oscilar no processo \\
Resistência & Está na pessoa & Aparece na relação \\
Função do terapeuta & Educar & Evocar/guiar \\
Objetivos da intervenção & Impostos & Negociados \\
\hline
\end{tabular}

Fonte: Adaptada de Emmons \& Rollnick, 2001; Miller \& Rollnick, 2002.

\section{Primeiros ingredientes da EM}

\section{Os cinco princípios gerais}

A atmosfera acolhedora descrita por Rogers, que envolve a empatia genuína (Rogers, 1959), pareceu ideal para estimular a reflexão da pessoa sobre suas próprias razões para mudar. A empatia foi então definida como elemento fundamental da EM, tornando-se parte dos princípios gerais da abordagem. A partir disso, diferentes teorias foram utilizadas para se pensar os demais princípios, o que também garantiu o embasamento da EM em evidências científicas da época. O modelo de dissonância cognitiva, por exemplo, proposto por Leon Festinger em 1957, foi utilizado como base para o princípio de desenvolver discrepância, processo utilizado desde os primórdios da abordagem motivacional. Desta forma, o reconhecimento da pessoa sobre a inconsistência entre o seu comportamento e seus valores, atitudes, objetivos ou sentimentos seria uma condição favorável à busca da consonância ou prontidão para mudar. O fenômeno da dissonância, de acordo com os estudos de Festinger, é "psicologicamente incômodo" e promove a busca pela resolução do conflito (Festinger, 1957; Draycott \& Dabbs, 1998).

Evitar argumentação e acompanhar a resistência foram incluídos nos princípios indicando a importância da não-confrontação. Por vezes traduzidos em um único princípio, estes referem-se ao cuidado do terapeuta em evitar convencer a pessoa a mudar (Miller \& Rollnick, 1991). Além disso, para tomar a decisão a pessoa precisa sentir-se capaz. O conceito de autoeficácia, introduzido por Albert Bandura na década de 1970, defende que as expectativas que uma pessoa possui sobre sua capacidade de realizar a mudança favorece a mudança propriamente dita (Bandura, 1977). Adaptado à EM, o princípio de promover a autoeficácia refere-se ao fortalecimento das percepções da pessoa quanto à sua capacidade de enfrentar obstáculos e ter êxito na mudança (Miller, 1983). Portanto, até 2008, os princípios gerais da EM incluíram (1) Expressar empatia; (2) Desenvolver discrepância; (3) Evitar argumentação; (4) Seguir com a resistência; e (5) Apoiar a autoeficácia. Mais tarde, estes princípios foram reformulados, porém foi mantida sua essência.

Motivação, ambivalência e os estágios de mudança

Com as dificuldades observadas por especialistas diante da mudança de comportamentos, na época da criação da EM alguns conceitos foram revistos ou introduzidos. A motivação, por exemplo, antes era concebida como um traço de caráter, passa a ser entendida como um estado, passível de modificação. A EM reforça este conceito e introduz, nesta dinâmica, a ambivalência como alvo de intervenção. Definida como um estado mental no qual uma pessoa tem sentimentos coexistentes, porém conflitantes a respeito de alguma coisa, a ambivalência reflete oscilações motivacionais normais que são passíveis de serem trabalhadas (Miller \& Rollnick, 1991). Ainda neste contexto, o Modelo Transteórico de Mudança (MT'T), introduzido em 1982 por Prochaska e DiClemente, representa uma referência teórica especialmente útil. Mais conhecido como os estágios de mudança, o MTT descreve de forma objetiva atitudes e dificuldades específicas de cada nível de motivação. A criação do MTT buscou descrever os principais estágios 
pelos quais as pessoas transitam durante a mudança de comportamento, independente da abordagem terapêutica utilizada (Prochaska \& DiClemente, 1983; Szupszynski \& Oliveira, 2008), pressupondo cinco estágios hierárquicos: pré-contemplação, caracterizado pelo não-reconhecimento do problema e não-intenção de mudança; contemplação, caracterizado pela coexistência de sentimentos opostos com relação a mudança, porém já existe um reconhecimento do problema; preparação, referindo-se ao planejamento de medidas a serem colocadas em prática para a mudança; ação, que refere-se à execução do plano de ação e atitudes em direção à mudança; e, finalmente, manutenção, compreendendo a continuidade de ações para manter a mudança alcançada.

O MTT reconhece fatores objetivos e subjetivos implicados na mudança de comportamento, por isso possui integração satisfatória com outras teorias (Prochaska \& DiClemente, 1983; Oliveira, Laranjeira, Araújo, Camilo, \& Schneider, 2003). Apesar da confusão existente entre as duas teorias, esclarece-se aqui que os estágios do MTT ofereceram uma maneira lógica de pensar o uso da EM e, em troca, a EM ofereceu um exemplo claro de como os profissionais podem ajudar a estimular a transição entre esses estágios, sendo consideradas, portanto, teorias complementares.

\section{As fases do processo de mudança}

O processo de mudança que ocorre durante a EM foi dividido, inicialmente, em dois sendo a primeira fase associada ao objetivo principal de estimular a motivação intrínseca para a mudança (Miller \& Rollnick, 1991). Nesta fase busca-se auxiliar a pessoa a responder a pergunta: "por que mudar?". O foco do trabalho aqui é identificar as motivações da pessoa e auxiliá-la a lidar com a ambivalência. Avançando para a segunda fase, o objetivo torna-se auxiliar a pessoa a responder a pergunta: “como mudar?", ou seja, tendo tomado uma decisão de mudar, o objetivo é reforçar o compromisso da pessoa e estabelecer um plano para alcançar a mudança.

\section{Estratégias iniciais}

As estratégias inicialmente formuladas para a EM foram mantidas, posteriormente organizadas no acrônimo OARS. Mais recentemente traduzido como PARR (Figlie \& Guimarães, 2014), o acrônimo refere-se às iniciais do inglês open questions, affirmations, reflective listening e summaries, que seriam, respectivamente, perguntas abertas, afirmações sobre as verbalizações da pessoa, escuta reflexiva e resumos daquilo que a pessoa traz (ver tabela 2).

Tabela 2. Descrição das principais estratégias da EM

\begin{tabular}{|c|c|c|}
\hline ESTRATÉGIA & DEFINIÇÃO & FUNÇÃO \\
\hline Open questions (perguntas abertas) & $\begin{array}{l}\text { Não usar perguntas fechadas com } \\
\text { respostas limitadas a "sim" ou } \\
\text { "não" }\end{array}$ & $\begin{array}{l}\text { Ajudar a pessoa a falar mais livremente, sendo } \\
\text { que sua fala é a ferramenta de trabalho principal } \\
\text { do terapeuta }\end{array}$ \\
\hline Affirmations (afirmativas) & $\begin{array}{l}\text { Comentários do terapeuta de } \\
\text { apreciação sobre as qualidades } \\
\text { da pessoa, pontos que podem } \\
\text { fortalecer sua autoeficácia }\end{array}$ & $\begin{array}{l}\text { Ver no outro seu melhor, nomeá-lo e ajudá-lo a } \\
\text { reconhecer suas qualidades também }\end{array}$ \\
\hline Reflective Listening (escuta reflexiva) & $\begin{array}{l}\text { Habilidade fundamental para se } \\
\text { estruturar a conversa. Pressupõe } \\
\text { empatia, respeito e compreensão } \\
\text { sobre a fala da pessoa e a no- } \\
\text { ção de que ela sabe mais do que } \\
\text { sabemos sobre suas dificuldades e } \\
\text { motivações }\end{array}$ & $\begin{array}{l}\text { Criar um clima propício para a conversa direcio- } \\
\text { nada para a mudança, devolver para a pessoa na } \\
\text { forma de reflexão, auxiliando a pessoa a se orga- } \\
\text { nizar e refletir sobre seus pensamentos, criando } \\
\text { uma narrativa coerente }\end{array}$ \\
\hline
\end{tabular}




\section{Evolução da EM: principais mudanças}

\section{O espírito da EM}

O espírito da EM se refere à postura do terapeuta, sobre a qual os princípios e estratégias se apoiam. $\mathrm{Na}$ segunda edição, este aspecto foi organizado em três elementos: (1) a colaboração, sugerindo a criação de uma parceria com a pessoa, honrando o seus desejos e perspectivas, e evitando confrontar; (2) a evocação, ou seja, buscar recursos e motivações da própria pessoa para a mudança; e (3) a autonomia, como reconhecimento do direito e capacidade da pessoa sobre suas escolhas. $\mathrm{Na}$ terceira e última edição, foi introduzido a compaixão, significando a garantia dos interesses da pessoa, em que o terapeuta evita impor o seu ponto de vista, por mais "certos" que estes pareçam.

\section{Novos princípios básicos}

Em 2008, foram propostas também mudanças nos princípios inicialmente definidos com a introdução do acrônimo RULE, que compreende: (1) resistir ao reflexo corretivo (resist the righting reflex), ou seja, vigiarmos nossa tendência de corrigir a pessoa como forma de tentar protegê-la ou achando que sabemos o que é melhor; (2) entender a motivação (understand motivation), significando guiar a conversa de acordo com seu nível de motivação atual; (3) ouvir (listen), que refere-se à escuta não-punitiva e empática; e (4) fortalecer (enpower), referindo-se a estimular a autonomia e autoeficácia (Rollnick, Miller \& Butler, 2008).

\section{As fases do processo de mudança}

As fases do processo de mudança foram aperfeiçoadas, visto que as duas inicialmente propostas foram consideradas insuficientes para explicar o processo dinâmico, complexo e não linear da mudança. Desta forma, em 2012, quatro processos são propostos: (1) engajando, momento de promover a aderência da pessoa no processo, fazendo com que ela se sinta acolhida, compreendida e segura, que pode garantir também seu retorno; (2) focando, etapa necessária e contínua durante o trabalho com a EM para definir o foco da conversa. $\mathrm{Na}$ medida que o trabalho caminha, novos objetivos podem aparecer e auxiliar com o objetivo maior, o que implicaria em mudança de foco; (3) evocando, processo muito particular da EM, esta etapa representa o alvo da conversa sobre a mudança, em que se trabalha a ambivalência e estimula a prontidão para mudar. O profissional procura evocar/ estimular falas da pessoa para a mudança em contraponto com falas que sustentam o comportamento atual; e (4) planejando, que sugere a elaboração e implementação de um plano de ação, uma vez que a decisão de mudar foi tomada.

É importante ressaltar que as fases são sequenciais e sobrepostas, ou seja, enquanto uma depende do êxito da anterior, elas acontecem dentro de uma dinâmica sem fronteiras bem definidas.

\section{A fala sobre a mudança ("change talk")}

$\mathrm{O}$ conceito da fala sobre a mudança (no original "change talk"), que substitui o conceito inicial de afirmaçoos de auto motivação (Miller \& Rollnick, 2012), se refere às verbalizações e argumentos oferecidos pela pessoa a favor da mudança de seu comportamento problema. Considerando que a fala para a mudança pode apresentar diferentes componentes que refletem a importância da mudança para a pessoa, na EM ela foi dividida em duas categorias principais de fala: uma que indica a preparação e outra que sugere a ação.

Assim, as categorias de fala para a mudança foram resumidas no acrônimo DARN CAT, criado para orientar o profissional na identificação destes importantes elementos no discurso da pessoa. Em uma linguagem preparatória, por exemplo, teríamos falas referindo-se ao desejo (desire) ("Gostaria que as coisas fossem diferentes"), às habilidades (abilities) ("Eu consegui ficar alguns dias sem usar"), às razões (reasons) ("Se eu diminuir o uso terei dinheiro para pagar as contas"), e às necessidades (need) ("Meu médico disse que preciso parar de beber"). O próximo estágio teria relação com a prontidão para realizar a mudança e envolve uma linguagem mobilizadora indicada pelas letras CAT: compromisso (compromise) ("Eu vou tentar"), ativando (acting) ("Estou planejando não ir à festa"), e dando passos (taking steps) ("Comecei a frequentar um grupo de autoajuda").

Elaborado com base em pesquisas de psicolinguística de Amrhein, o conceito de fala para a mudança teoriza sobre a influência da linguagem natural no processo de decisão e comprometimento das pessoas. Estudos indicam que a linguagem de compromisso seria o elemento de maior impacto sobre a mudança de comportamento (Amrhein, 1992; Amrhein, Miller, Yahne, Palmer, \& Fulcher, 2003). Além disso, a fala em si não promove mudança se não for espontânea, ou seja, se a mesma não for gerada por motivações intrínsecas da pessoa. Neste sentido, a estimulação da introspecção e a geração de pensamentos e razões genuínas para a mudança promovida pela interação terapêutica (e não por simples verbalização 
ou repetição sobre a mudança) seria responsável pela ativação de áreas cerebrais específicas que conduzem à ação (Ewing, Yezhuvath, Houck \& Filbey, 2014).

Reformulando o conceito de resistência e a fala que sustenta o status quo ("sustain talk")

Ao longo da evolução da teoria motivacional, o conceito de resistência foi alvo de importantes revisões. Chamar de resistência algo que surge naturalmente ao falar sobre a mudança foi entendido como "patologizar" a reação da pessoa e desresponsabilizar o profissional. Desta forma, introduziu-se o conceito de fala que sustenta o status quo, ou sustain talk (originalmente chamada de afirmações antimotivacionais). Estas teriam relação com o "lado" da ambivalência que defende não mudar, que seria o oposto de fala sobre a mudança.

Por outro lado, a discordância seria a parte da resistência, que diz respeito à interação, indicando a falta de sintonia entre o profissional e o cliente/paciente. Portanto, a fala que sustenta o comportamento atual tem relação com argumentos da pessoa a favor do comportamento-problema e discordância é o que pode surgir durante a conversa quando a pessoa não concorda com afirmações do profissional. Uma forma de trabalhar com isso é utilizar reflexões, que promovem a escuta da pessoa sobre suas próprias verbalizações e pode auxiliá-la a repensar seus argumentos a favor do status quo.
Ver a tabela 3 para um resumo das principais mudanças realizadas ao longo das três revisões teóricas da EM.

\section{Adaptações do método motivacional}

$\mathrm{Na} 2^{\mathrm{a}}$ edição do livro, Miller e Rollnick dedicam um capítulo ao que eles denominaram adaptações da entrevista motivacional (adaptation of motivational interviewing - AMI). As AMI referem-se à combinações dos princípios básicos da EM com ferramentas terapêuticas diversas. O exemplo pioneiro disso é o chamado drinker's check-up (DCU) (Miller, Sovereign \& Krege, 1988). Este modelo de intervenção breve, criado a partir da EM, integra uma avaliação do padrão de uso de substâncias à devolutiva sobre seu resultado (feedback), realizada a partir de princípios da EM. Resultados de estudos sobre o DCU são animadores com relação ao aumento da motivação para mudar, ao engajamento ao tratamento e à redução do uso (Miller, Sovereign \& Krege, 1988; Hester, Squires, \& Delaney, 2005; Carey, Balestrieri, Miller, Merrill, DiBello, \& Benz, 2017).

Com base neste modelo, alternativas de avaliação seguidas de feedback baseadas na EM foram ampliadas. Assim, a EM como estratégia de feedback personalizado a partir de resultados de avaliação passou a ser conhecida como Motivational Enhancement Therapy (MET) (Miller, Zweben, DiClemente \& Rychtarik, 1992). Um

Tabela 3. Principais mudanças nas obras de referência da Entrevista Motivacional

\begin{tabular}{|c|c|c|}
\hline $1^{a}$ edição & $2^{a}$ edição & $3^{a}$ edição \\
\hline $\begin{array}{l}\text { Publicada originalmente em } \\
\text { inglês em 1991, traduzida e pu- } \\
\text { blicada no Brasil em } 2001\end{array}$ & $\begin{array}{l}\text { Publicada originalmente em inglês no ano } \\
\text { de } 2002 \text {, nunca traduzida para o Português }\end{array}$ & $\begin{array}{l}\text { Publicada originalmente em inglês em } \\
\text { 2012, nuca traduzida para o Português }\end{array}$ \\
\hline $\begin{array}{l}\text { Foco em comportamentos } \\
\text { aditivos }\end{array}$ & $\begin{array}{l}\text { Direcionada para mudanças de comporta- } \\
\text { mento diversos }\end{array}$ & $\begin{array}{l}\text { Amplia a utilidade da EM para mudanças } \\
\text { diversas }\end{array}$ \\
\hline $\begin{array}{l}\text { Processo de mudança estrutura- } \\
\text { do em duas fases: } 1^{\mathrm{a}} \text { Estimular a } \\
\text { motivação para a mudança e } 2^{\mathrm{a}} \\
\text { Fortalecer o compromisso com } \\
\text { a mudança }\end{array}$ & $\begin{array}{l}\text { Processo de mudança continua sendo traba- } \\
\text { lhado em duas fases }\end{array}$ & $\begin{array}{l}\text { O processo passa a ser estruturado em } \\
\text { quatro etapas: engajar, focar, evocar e } \\
\text { planejar }\end{array}$ \\
\hline $\begin{array}{l}\text { Ferramenta principal: os } 5 \text { prin- } \\
\text { cípios básicos }\end{array}$ & $\begin{array}{l}\text { Ferramenta OARS e dar informação/con- } \\
\text { selho }\end{array}$ & \\
\hline $\begin{array}{l}\text { Conceito de resistência como } \\
\text { parte integrante da obra }\end{array}$ & $\begin{array}{l}\text { Abandono do conceito de resistência e } \\
\text { adoção dos conceitos de "sustain talk" e } \\
\text { "change talk" }\end{array}$ & $\begin{array}{l}\text { O conceito de resistência é considerado } \\
\text { como uma alternância entre a fala que } \\
\text { sustenta o 'status quo' e discordância do } \\
\text { terapeuta }\end{array}$ \\
\hline
\end{tabular}


exemplo importante disso foi o projeto MATCH, um amplo estudo clínico conduzido para avaliar a eficácia de tratamento psicossocial no tratamento de alcoolistas (Project MATCH Research Group, 1997). Além disso, nos contextos de atenção primária, o SBIRT (Screening/avaliação, Brief Intervention/intervenção breve e Referral to Treatment/encaminhamento para tratamento) representa atualmente uma ferramenta importante para casos em que o uso de substâncias é identificado. O mesmo baseia-se fundamentalmente na identificação do risco relacionado ao uso seguido de intervenção breve motivacional (Babor, McRee, Kassebaum, Grimaldi, Ahmed, \& Bray, 2007).

Outros modelos incluem a EM como pré-tratamento, suplemento ou integrada a outras terapias (Arkowitz \& Miller, 2011). Os modelos mais explorados na literatura neste caso são as combinações da EM com a Terapia Cognitivo-comportamental (TCC), aplicados ao uso de substâncias assim como a outros diversos problemas comportamentais. Assim, com a característica adaptável do método, a EM passa a ser testada com transtornos alimentares, depressão e ansiedade (Carroll, Ball, Nich, Martino, Frankforter, Farentinos, Kunkel, Mikulich-Gilbertson, Morgenstern, Obert, Polcin, Snead, \& Woody, 2006; Arkowitz, Westra, Miller, \& Rollnick, 2011; Klimas, Tobin, Field, O'Gorman, Glynn, Keenan, Saunders, Bury, Dunne \& Cullen, 2014; Barrera, Smith, \& Norton, 2016; Gates, Sabioni, Copeland, Le Foll, \& Gowing, 2016), questões de saúde como dietas, atividade física, controle da diabetes e adesão medicamentosa (Christie \& Channon, 2014; Lewis-Fernandéz, Coombs, Balán, \& Interian, 2018), além de populações específicas, como no caso de adolescente (Andretta \& Oliveira, 2011).

\section{Discussão}

A EM introduz, no contexto de atenção aos problemas relacionados ao uso de substâncias, um estilo de comunicação diferenciado para que a pessoa alcance suas próprias conclusões sobre a importância da mudança. Para isto, desde o início a EM foi pensada com base em princípios e estratégias muito particulares. Mudanças na forma como os primeiros elementos da EM foram concebidos são entendidas como parte importante da evolução do método e de esclarecimentos que foram se fazendo necessários no decorrer dos anos que seguiram sua criação. No entanto, a prática e as pesquisas sobre a EM no Brasil parecem ainda pautar-se na teoria como ela foi construída inicialmente.

De maneira geral, a EM tem se mostrado efetiva para promover a mudança de comportamento. Uma revisão recente com 34 ensaios clínicos realizados nos últimos 10 anos apontou resultados positivos da EM quando aplicada ao tratamento dos transtornos por uso de substâncias (DiClemente, Corno, Graydon, Wiprovnick, \& Knoblach, 2017), enquanto que no Brasil, uma revisão realizada não foi conclusiva com relação aos efeitos da EM, principalmente por esta forma de tratamento estar muito associada a outras abordagens (Andretta, Meyer, Kuhn, \& Rigon 2014). Variações entre os resultados são justificadas principalmente nas variações metodológicas, como número variado de sessões e amostras diversas, além da qualidade da intervenção (Andretta, Meyer, Kuhn \& Rigon, 2014; Romano \& Peters, 2016; Magill, Apodaca, Borsari, Gaume, Hoadley, Gordon, Tonigan, \& Moyers, 2018).

Reconhecida como abordagem de escolha para utilizar com pessoas que precisam realizar mudanças em algum aspecto de sua vida, a importância da EM parece aumentar considerando os crescentes conflitos gerados com os atrativos da vida atual (redes sociais, jogos, compras, comida, etc.), muitas vezes associados a prejuízos diversos. Todos estão sujeitos a precisar repensar os comportamentos e a buscar fazer algo a respeito quando o mesmo está dissonante (ou discrepante) com os objetivos maiores e valores. Neste sentido, estratégias da EM podem ser muito eficazes para o processo de decisão de uma pessoa, fortalecendo sua motivação para se comprometer e mudar.

\section{Referências}

Amrhein, P.C. (1992). The comprehension of quasi-performative verbs in verbal commitments: New evidence for componential theories of lexical meaning. Journal of Memory and Language, 31(6), 756-784.

Amrhein, P.C., Miller, W.R., Yahne, C.E., Palmer, M., \& Fulcher, L. (2003). Client commitment language during motivational interviewing predicts drug use outcomes. Journal of Consulting and Clinical Psychology, 71(5), 862-878.

Andretta, I., Meyer, E., Kuhn, R.P., \& Rigon, M. (2014). A entrevista motivacional no Brasil: uma revisão sistemática. Mudanças - Psicologia da saúde, 22(2), 15-21.

Andretta, I. \& Oliveira, M.S.(2011). A entrevista motivacional em adolescentes usuários de droga que cometeram ato infracional. Psicologia: Reflexão e Critica, 24(2), 218-226.

Arkowitz, H., \& Miller, W.R. (2011). Aprendizado, aplicação e extensão da entrevista motivacional. In: Arkowitz, H., Westra, H.A., Miller, W.R., \& Rollnick, S., organizadores. Entrevista Motivacional no tratamento de problemas psicológicos. p. 1-21. São Paulo: Roca.

Arkowitz, H., Westra, H.A., Miller, W.R., \& Rollnick, S. (2011). Entrevista Motivacional no tratamento de problemas psicológicos. São Paulo: Roca.

Babor, T.F., McRee, B.G., Kassebaum, P.A., Grimaldi, P.L., Ahmed, K., \& Bray, J. (2007). Screening, Brief Intervention, and Referral to Treatment (SBIRT): Toward a Public Health Approach to the Management of Substance Abuse. Substance Abuse,_28(3), 7-30.

Bandura, A. (1977). Self-efficacy: Towards a unifying theory of behavior change. Psychological Review, 84(2), 191-215.

Barrera, T.L., Smith, A, H., \& Norton, P.J. (2016). Motivational Interviewing as an Adjunct to Cognitive Behavioral Therapy for Anxiety. Journal of Clinical Psychology, 72(1), 5-14. 
Carey, K.B., Balestrieri, S.G., Miller, M.B., Merrill, J.E., DiBello, A.M., \& Benz, M.B. (2017). Efficacy of the College Drinkers Check-Up for student drinkers living off campus. Journal of Studies on Alcohol and Drugs,78(4), 571-579.

Carroll, K.M., Ball, S.A., Nich, C., Martino, S., Frankforter, T.L., Farentinos, C., Kunkel, L.E., Mikulich-Gilbertson, S.K., Morgenstern, J., Obert, J.L., Polcin, D., Snead, N., \& Woody, G.E.; National Institute on Drug Abuse Clinical Trials Network. (2006). Motivational interviewing to improve treatment engagement and outcome in individuals seeking treatment for substance abuse: a multisite effectiveness study. Drug and Alcohol Dependence, 81(3), 301-312.

Christie, D., \& Channon, S. (2014). The potential for motivational interviewing to improve outcomes in the management of diabetes and obesity in pediatric and adult populations: a clinical review. Diabetes, Obesity and Metabolism,16(5), 381-387.

Covell, N.H., McCorkle, B.H., Weismann, E.M., Summerfelt, T., \& Essock, S.M. (2007). What's in a name? Terms preferred by service recipients. Administration and Policy in Mental Health and Mental Health Services Research, 34(5), 443-7.

DiClemente, C.C., Corno, C.M., Graydon, M.M., Wiprovnick, A.E., \& Knoblach, D.J. (2017). Motivational Interviewing, Enhancement, and Brief Interventions Over the Last Decade: A Review of Reviews of Efficacy and Effectiveness. Psychology of Addictive Behaviors, 31(8), 862- 887.

Draycott, S., \& Dabbs, A. (1998). Cognitive dissonance. A theoretical grounding of motivational interviewing. British Journal of Clinical Psychology, 37(Pt 3), 355-364.

Emmons, K.M., \& Rollnick, S. (2001). Motivational interviewing in health care settings. Opportunities and limitations. American Journal of Preventive Medicine, 20(1), 68-74.

Ewing, S.W.F., Yezhuvath, U., Houck, J.M., \& Filbey, F.M. (2014). Brain-based origins of change language: a beginning. Addictive Behaviors, 39(12), 1904 1910.

Festinger, L. (1957). A theory of cognitive dissonance. Stanford, CA: Stanford University Press.

Figlie, N.B., \& Guimaraes, L.P. (2014). A Entrevista Motivacional: conversas sobre mudança. Boletim da Academia Paulista de Psicologia, 34(87), 472-489.

Gates, P.J., Sabioni, P., Copeland, J., Le Foll, B., \& Gowing, L. (2016). Psychosocial interventions for cannabis use disorder. Cochrane Database of Systematic Reviews, (5), CD005336.

Hester, R.K., Squires, D.D., \& Delaney, H.D. (2005). The Drinker's Check-up: 12-month outcomes of a controlled clinical trial of a stand-alone software program for problem drinkers. Journal of Substance Abuse Treatment, 28(2), 159-69.

Klimas, J., Tobin, H., Field, C.A., O’Gorman, C.S.M., Glynn, L.G., Keenan, E., Saunders, J., Bury, G., Dunne, C., \& Cullen, W. (2014). Psychosocial interventions to reduce alcohol consumption in concurrent problem alcohol and illicit drug users. Cochrane Database of Systematic Reviews, (12): CD009269.

Lewis-Fernandéz, R., Coombs, A.A., Balán, I.C., \& Interian, A. (2018). Motivational Interviewing: Overcoming Disparities in Pharmacotherapy Engagement. Journal of Clinical Psychiatry, 79(3), pii: 18 ac12150.
Magill, M., Apodaca, T.R., Borsari, B., Gaume, J., Hoadley, A., Gordon, R.E.F., Tonigan, J.S., \& Moyers, T. (2018). A meta-analysis of motivational interviewing process: Technical, relational, and conditional process models of change. Journal of Consulting and Clinical Psychology, 86(2), 140-157.

Miller, W.R. (1983). Motivational interviewing with problem drinkers. Bebavioural and Cognitive Psychotherapy, 11, 147-172.

Miller, W.R., \& Rollnick, S. (1991). Motivational interviewing: Preparing people to change addictive behavior. New York: Guilford Press.

Miller, W.R., \& Rollnick, S. (2002). Motivational interviewing: Preparing people for change. 2nd ed. New York: Guilford Press.

Miller, W.R., \& Rollnick, S. (2009). Ten things that motivational interviewing is not. Behavioural and Cognitive Psychotherapy, 37(2), 129-140.

Miller, W.R., \& Rollnick, S. (2012). Motivational Interviewing. In: Helping People Change. 3rd ed. New York: Guilford Press.

Miller, W.R., \& Rose, G.S. (2009). Toward a theory of motivational interviewing. American Psychology, 64(6), 527-537.

Miller, W.R., Sovereign, R.G., \& Krege, B. (1988). Motivational interviewing with problem drinkers: II. The Drinker's Check-up as a preventive intervention. Behavioural and Cognitive Psychotherapy, 16(4), 251-268.

Miller, W.R., Zweben, A., DiClemente, C.C., \& Rychtarik, R.C. (1992). Motivational Enhancement Therapy manual: A clinical research guide for therapists treating individuals with alcohol abuse and dependence. Project MATCH Monograph Series, Vol. 2. Rockville, Maryland: National Institute on Alcohol Abuse and Alcoholism.

Moyers, T.B., \& Rollnick, S. (2002). A motivational interviewing perspective on resistance in psychotherapy. Journal of Clinical Psychology, 58(2), 185-193.

Oliveira. M., Laranjeira, R., Araújo, R.B., Camilo, R.L., \& Schneider, D.D. (2003). Estudos dos estágios motivacionais em sujeitos adultos dependentes de álcool. Psicologia: Reflexão e Critica, 16(2), 265-270.

Prochaska, J.O., \& DiClemente, C.C. (1983). Stages and processes of self-change of smoking: toward an integrative model of change. Journal of Consulting and Clinical Psychology, 51(3), 390-395.

Project MATCH Research Group (1997). Matching alcoholism treatments to client heterogeneity: Project MATCH posttreatment drinking outcomes. Journal on Studies of Alcohol and Drugs, 58(1), 7-29.

Rogers, C.R. (1951). Client-centered therapy. Boston: Houghton-Mifflin.

Rogers, C.R. (1959). A theory of therapy, personality, and interpersonal relationships as developed in the client-centered framework. New York: McGraw-Hill.

Rollnick. S., Miller, W.R., \& Butler, C.C. (2008). Motivational interviewing in health care: Helping patients change behavior. New York: Guilford Press.

Romano, M. \& Peters, L. (2016). Understanding the process of motivational interviewing: A review of the relational and technical hypotheses. Psychotherapy Research, 26(2), 220-240.

Szupszynski, K.P.D.R., \& Oliveira, M.S. (2008). O Modelo Transteórico no tratamento da dependência química. Psicologia: Teoria e Prática, 10(1), 162-173.

White, W.L., \& Miller, W.R. (2007). The use of confrontation in addiction treatment: History, science, and time for change. Counselor, 8(4), 12-30.

Submetido em: 8-12-2019

Aceito em: 17-12-2019 